\title{
Green Backhauling for Heterogeneous Mobile Access Networks: What Are the Challenges?
}

\author{
Farias, F.S. ', P. Monti ${ }^{2}$, A. Văstberg ${ }^{2}$, M. Nilson ${ }^{2}$, Costa, J. C. W. A.', L. Wosinska ${ }^{2}$ \\ 'Universidade Federal do Pará, Belém, Brazil \\ ${ }^{2}$ KTH Royal Institute of Technology, Kista, Sweden \\ fabriciosf@ufpa.br, pmonti@kth.se, vastberg@kth.se,matsnils@kth.se, jweyl@ufpa.br,wosinska@kth.se
}

\begin{abstract}
Abstraci-Heterogeneous network (HetNet) deployment strategies have the potential to improve the energy efficiency of mobile access networks. One key aspect to consider in HetNets is the impact of the power consumption of the backhaul, i.e., the overall energy efficiency of a HetNet deployment is affected by the backhaul technology and architecture. This paper presents a preliminary assessment of the design challenges of a future green backhaul segment for a HetNet deployment. The study is based on the analysis of the medium term future outlook (i.e., between now and the year 2025) of the main technologies used in todays' backhaul networks (i.e., fiber, microwave and copper). It can be concluded that, even if there are no doubts that both microwave and fiber will be predominately used in the future, the possible migration paths leading to such fiber-and microwave-dominated scenarios might be different, depending on factors such as spectrum and license costs, time to deployment, availability of equipment, and required Quality of Service (QoS) Ievels.
\end{abstract}

Keywords: HetNet, energy efficiency, migration strategies, macro, pico, micro, femto, mobile backhual, fiber, microwave, copper.

\section{INTRODUCTION}

One way of reducing the power consumption in wireless mobile networks is to use heterogeneous network (HetNet) deployment strategies. The key rationale is to tailor the network deployment to the expected traffic load, i.e., to provide coverage via macro base stations and to guarantee high capacity only where it is needed via micro, pico, and/or femto cells. Thereby the number of power hungry base stations needed, and consequently the overall energy consumption may be reduced. In mobile networks the backhaul contribution to the total power consumption is usually neglected because of its limited impact compared to that of the radio base stations. However, meeting the almost exponential increase in mobile data traffic [1-4] requires a large number of (mainly small) base stations. It has been shown that the total power consumption of a HetNet deployment to a larger degree is affected not only by the presence of the backhaul [5] but also by its specific technological and architectural choices [6]. This means that backhaul networks will take a significant share of the cost and the energy consumption in future systems, and also that their actual contribution to the energy consumption will depend on the base station deployment scenario as well as on the technology and architectural choice for the backhaul itself. This said it is of the outmost importance to have a holistic understanding of how to achieve a green backhaul segment for future HetNet deployments.

The work presented in this paper was partly supported by GreenHaul a Wireless(a)KTH seed project (Call 12/2. Pr. \#63188), CAPES and CNPQ/Brazil.
Currently backhaul is to a large extent based on microwave, copper and fiber. These technologies are complementary and offer different advantages depending on the deployment scenario. Fiber-based alternatives come today at a relatively high deployment cost (CAPEX) but offer long-term support with respect to increasing capacity demand [7]. A microwavebased backhaul is attractive in terms of short time-to-market, low investment in infrastructure and simple deployment [8]. Digital Subscriber Lines (DSLs) might be still appealing in the presence of an existing copper infrastructure, bearing in mind their capacity limitations [9]. On the other hand, what will be the challenges in designing a future (i.e., in ten years from now) green backhaul segment and what will be the role of each one of these technologies is still an open question.

This paper aims at providing a preliminary study to answer these questions. The presented assessment is one of the first in its kind because it combines various technology specific aspects including energy performance, time to deployment, capacity and transmission ranges, in addition to CAPEX and OPEX considerations. As part of the study we consider two HetNet deployment scenarios with the corresponding backhaul. The first one represents a dense urban area with traffic levels comparable to the ones expected in the short term future (i.e., year 2015). The second one is a snapshot of the same urban area, but in year 2025. The medium term future outlook (i.e., between now and year 2025) of fiber, microwave and copper is then analyzed to understand the possible migration scenarios and their main drivers that will take us from the current backhaul deployment scenarios to the one envisioned in ten years from now.

The assessment makes it clear that it is not possible to find a "one size fits all" migration solution. Even if there are no doubts that both microwave and fiber will be predominant in future backhaul networks, the possible migration paths leading to such scenarios might vary based on a number of factors, such as the presence of an existing infrastructure; spectrum and license costs; availability of equipment; willingness to invest in a completely new infrastructure; technology time to deployment; and, finally, the Quality of Service (QoS) levels to be provided to the end user.

\section{HETEROGENEOUS NETWORKS: BASE STATION TYPES AND BACKHAUL TECHNOLOGY OPTIONS}

The objective of this section is to provide a description of $(i)$ the base station types used in a HetNet deployment, and (ii) the technological options available for the backhaul segment. 


\section{A. HetNet base station types}

This section describes the various base station types (i.e., macro, micro, pico, and femto) that are usually part of a HetNet deployment. Their main characteristics are summarized in Table 1 [10-11].

TABLE I. BASE STATION TYPES AND CHARACTERISTICS

\begin{tabular}{|c|c|c|c|}
\hline \multirow{2}{*}{ Type } & \multirow{2}{*}{ Coverage } & \multicolumn{2}{|c|}{ Radiated Power $[\mathbf{W}]$} \\
\cline { 3 - 4 } & $<35 \mathrm{~km}$ & Outdoor & Indoor \\
\hline Macro & $<-40$ & - \\
\hline Micro & $<2 \mathrm{~km}$ & $0.5-2$ & - \\
\hline Pico & $<200 \mathrm{~m}$ & $0.25-2$ & 0.1 \\
\hline Femto & $10-15 \mathrm{~m}$ & - & $<0.1$ \\
\hline
\end{tabular}

Macro base stations (deployed outdoor, over-rooftop level) are able to cover a vast area and to support a very high number of users. Capital expenditures (CAPEX) and operation expenditures (OPEX) are high due to their price, their site acquisition/rental costs, and their energy consumption levels. Macro base stations can provide $2 \mathrm{G}, 3 \mathrm{G}$ and $4 \mathrm{G}$ services. Legacy $2 \mathrm{G}$ (i.e., GSM, CDMA-IS95) sites are usually backhauled using copper, while 3G (i.e., UMTS, CDMA2000) and newly deployed 4G (i.e., LTE, WIMAX) base station sites use fiber and/or microwave for backhaul. Micro base stations are also deployed outdoors, but below-rooftop levels (e.g., lamp posts, external wall) to cover a specific area (e.g., street, block). Their size, cost and radiated power levels are lower compared to macro base stations. They are usually backhauled via microwave. Pico base stations have lower coverage than macro and micro and consequently need a lower transmission power. They are deployed often to remedy the coverage/capacity holes in a given area, i.e., hot-spots. When deployed indoor, pico base station are usually backhauled through an existing broadband infrastructure, provided it has enough capacity (i.e., fiber to the home/curb combined with Ethernet), while outdoors deployed pico base stations are mostly backhauled via microwave. Femto are a completely different type of base station. While macro, micro and pico are industrial grade equipment (i.e., higher performance level and price), femto base stations are meant for the consumer market. They are typically deployed in indoor residential and business premises. Their role is similar to pico base stations, i.e., to provide extra capacity where needed, but their power consumption is lower due to their proximity to the user and their short coverage radius (i.e., limited to a few tens of meters). They are usually backhauled via the users existing broadband infrastructure, i.e., digital subscriber line (DSL), cable modem, Ethernet, or fiber.

\section{B. Backhaul access technology for HetNets}

There are several technologies that can be used to backhaul the aggregated traffic coming from a HetNet deployment. Among the various options, fiber, copper, and microwave are the most popular choices [12].

Fiber can provide virtually unlimited capacity thus guaranteeing the backhaul segment to sustain any future increase of bandwidth requirement, especially in dense urban areas [3]. On the other hand, reaching an adequate fiber infrastructure deployment level can take many years [9], while the dramatic increase of backhaul traffic is already becoming an issue [2]. For this reason alternative backhaul access mediums (i.e., microwave and copper) also play an important role.

Microwave is widely used in both urban and rural regions [8][13], mostly because of the low deployment cost. On the other hand backhaul solutions based on microwave force operators to lease spectrum resources, which can bring a significant extra cost depending on the amount of spectrum resources used. Microwave backhauling can be divided into three categories [14]. The first one is Microwave Point To Point (PTP). PTP requires a dedicated link (in the $2-30 \mathrm{GHz}$ range) to connect each Radio Access Network (RAN) site to a hub node that is in turn connected to the metro/aggregation segment. If the RAN site is too far from the hub, or if there is no Line of Sight (LOS) connectivity, the backhaul may include multiple hops [6]. The second category of microwavebased backhaul solutions is based on E-band PTP links. This backhaul solution has the same characteristics (i.e., in terms of topology options) of the one based on conventional microwave PTP links, but it uses a different spectrum range, i.e., from 70 to $80 \mathrm{GHz}$. Because of the higher frequency a E-band PTP backhaul has a shorter reach and its performance might be severely affected by environmental factors, e.g., rain. The last category of microwave-based backhaul solutions is based on Microwave Point to Multiple Point (PMP) links. With this option one access point (AP) in the hub can connect simultaneously to multiple RAN sites thus reducing the number of required dedicated links, leading to an obvious saving in terms of the radio equipment.

Copper is the legacy technology used in the past decades to provide fixed broadband connectivity (i.e., ADSL, VDSL, etc.) and to backhaul traffic in the early generations of mobile services (i.e., 2G and 3G). The main drawback of this technology is its inability to provide high capacities over long distances (e.g., VDSL2 bonding can provide up to $100 \mathrm{Mbit} / \mathrm{s}$ downstream and up to $5,4 \mathrm{Mbit} / \mathrm{s}$ upstream over 1500 meters [7]). The latest improvement in DLS technology (i.e., G.Vector and G.Fast [9]) may soon enable rates up to $500 \mathrm{Mbits} / \mathrm{s}$ but still over very short distances. i.e., up to $100 \mathrm{~m}$. On the other hand, copper is already widely deployed making it still an attractive option for a number of backhauling scenarios especially in the short term future, while more capacity efficient backhaul solutions (i.e., based on fiber or microwave) are being deployed [8] to both enhance the existing copper based backhaul and to cater for the longer term traffic requirements. For example in a Fiber-to-the-Cabinet (FTTC) or Fiber-to-the-Building (FTTB) scenario mobile operators may still benefit from an already deployed copperbased last mile especially to backhaul small base stations (i.e., pico and femto) whose aggregated bandwidth is not huge. 
III. HETNET DEPLOYMENT WITH BACKHAUL IN A DENSE URBAN AREA: A REPRESENTATIVE SCENARIO

Based on the considerations made so far, this section aims at providing a snapshot (Figure 1) of how the deployment of a heterogeneous mobile wireless network (with backhaul) may look like in a dense urban area with today's short term (i.e., between year 2013 and 2015) traffic level requirements.

As it can be seen from the Figure 1, the scenario is mainly dominated by the deployment of outdoor base stations (i.e., macro and micro), in addition to a relatively low number of pico and femto base stations [1][4][7-9][13-15]. This is motivated by today's traffic levels that still do not justify a massive indoor base station deployment. In terms of backhaul, the figure shows how macro, micro, pico, and femto base stations can use copper, fiber and/or microwave, depending on the specific situation. For legacy $2 \mathrm{G}$ base stations, which carry mostly voice traffic, the backhaul is copper based. Copper is also used to backhaul residential customers and business premises that are already using VDSL2 lines [7], i.e., as a "last mile" in a more general FTTC scenario. FTTH solutions, on the other hand, are already available, but not widely deployed. Microwave is responsible to backhaul micro base stations arranged on street poles, while fiber backhaul is mostly used for macro base station.

The Figure 1 also presents interesting aspects of today's mobile access networks, with WLAN being used more widely than femto base stations and acting as offloading data traffic points. This will certainly benefit operators that can use the extra capacity elsewhere. On the other hand compared to femto base stations a mere WLAN access point does not have the same capabilities to deliver all requested services to the users (e.g., short message service, voice mail, calls, etc.). This is the main reasons why femto base stations are gaining popularity. predict possible evolution/migration paths for the backhaul it is important to first better understand the future technology outlook of fiber, microwave and copper, as explained in the next section.

\section{PERFOMANCE OF HETNETS: IMPACT OF THE BACKHAUL SEGMENT WITH FUTURE TECHNOLOGY OUTLOOK}

This section looks more into detail on how a specific technological and the architectural choice for the backhaul may impact the overall performance of a HzetNet-based wireless access deployment.

The main advantage of choosing HetNets over homogeneous networks is represented by the possible energy savings. On the other hand it was found [5] that with the deployment of a relative high number of base stations not only the power contribution of the backhaul cannot be neglected, but also it becomes a crucial aspect to consider. In such scenario the backhaul power consumption may vary depending on what kind of technology is being used. Among the various alternatives fiber is the most energy efficient [6]. However this does not necessarily mean that all current and future backhaul solutions should be based on a massive fiber deployment. There are in fact also other aspects that play an equally important role. Neglecting them may lead to backhaul solutions that are energy efficient, but suboptimal (or worse) with respect to other performance. More specifically it is also essential to consider: (i) the backhaul Capital and Operational Expenditures (CAPEX and OPEX), (ii) the provided capacity (i.e., how much bandwidth a specific technology can offer), (iii) the time to deployment of a certain technology; and (iv) the range, i.e., the maximum distance of a backhaul link that can be offered.

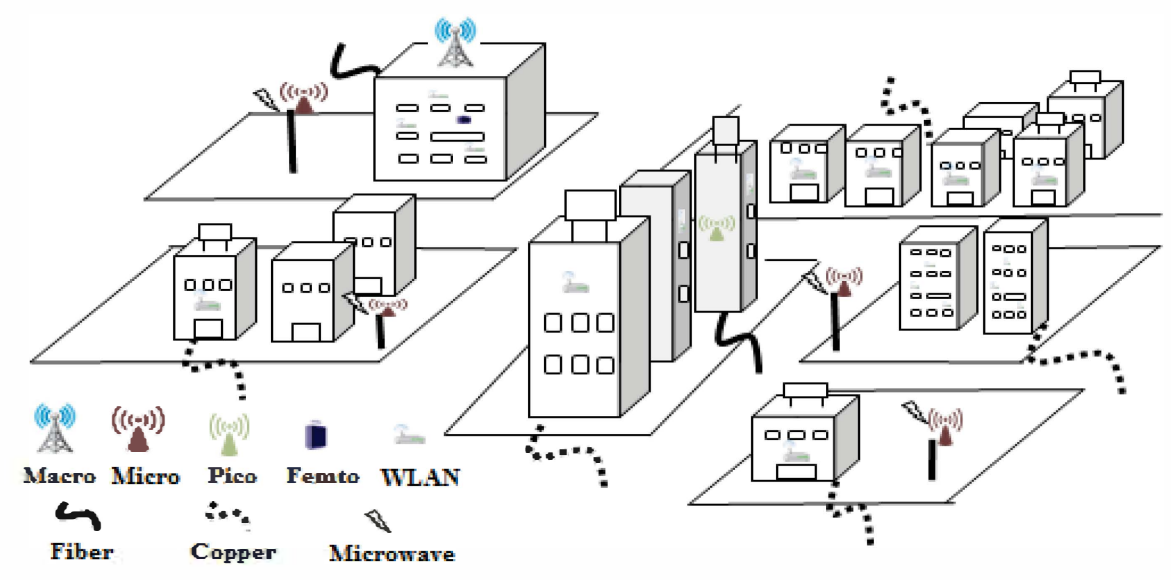

Figure 1. HetNet deployment with relative backhaul in a dense urban area.

The snapshot in Figure 1 is expected to change rapidly driven by an increasing in the traffic demand (estimated in 11,2 Exabyte's per month in the medium term future [4]). The mobile access segment will certainly evolve towards a more heterogeneous scenario (i.e., a larger indoor deployment of pico and femto base stations). On the other hand in order to
These parameters (together with the energy performance) are essential to understand the future outlook of the main backhaul technologies. Table Il summarizes this information considering two specific cases, i.e., five and ten year from now. From the table it becomes clear that there is no single technology able to outperform all the others in each and every 
aspect. All of them have a number of attractive properties and of course a few drawbacks.

More specifically, it can be seen that fiber is the most energy efficiency technology but it also has the longest time to deployment, as well as highest CAPEX, and OPEX [14]. The advantage that makes fiber an interesting alternative for backhaul is related to its almost unlimited capacity, long reach and low energy consumption. Today fiber provides backhauling mainly for macro base stations. However, new infrastructures are being deployed in order to bring fiber resources even closer to the user premises. In the next few years, it is expected to have fiber to the home or fiber to the building widely available in urban areas, thus enabling fiberbased backhaul solutions for small base stations (i.e., pico and femto). In the next seven to ten years the main expense for a fiber-based backhaul will shift from CAPEX to OPEX [14][15][16]. This is because once the fiber infrastructure is deployed money will be spent mostly on leasing fiber resources. three technologies, due to the equipment price. However, the OPEX is lower compared to the other technologies. On the other hand, microwave is not as good as fiber in terms of energy efficiency [6]. Another disadvantage of microwave is dependence on weather conditions (e.g., rain) that might affect the quality of the transmission.

The information included in Table II can also be used to make a number of educated guesses on how the scenario presented in Figure 1 will look like in ten years (i.e., in 2025). Considering that today the backhaul infrastructure in some countries (e.g., Germany) comprises $50 \%, 35 \%$ and $15 \%$ of microwave, copper, and fiber resources respectively [1], it is clear from the discussion above that a migration from copper to fiber and/or microwave is inevitable, on the other hand a few copper-based backhaul cases might still remain for mostly low capacity, low budget backhaul applications. More details are presented in the next section.

TABLE II. BACKHAUL TECHNOLOGIES VS. PERFORMNCE METRICS

\begin{tabular}{|c|c|c|c|c|c|c|}
\hline \multirow{2}{*}{ Metries } & \multicolumn{3}{|c|}{ Techology outlook } & \multicolumn{3}{c|}{ Next 10 Years } \\
\cline { 2 - 7 } & Fiber & Copper & Microwave & Fiber & Copper & Microwave \\
\cline { 2 - 7 } & Excellent & Average & Good & Excellent & Average & Good \\
\hline Energy Efficiency & Very high & Not necessary & High & Average & High & Average \\
\hline CAPEX & Average & Average & Low & Very High & Very High & Low \\
\hline OPEX & Very high & Medium & High & Very high & Low & High \\
\hline Capacity & Very high & Immediate & Short & High & Short & Short \\
\hline Time to Deploy & Very high & Very short & Medium & High & Very Shor & Medium \\
\hline Range & & & & \multicolumn{2}{c}{}
\end{tabular}

Copper is currently available, mostly through VDSL2, in almost all user and business premises. This means that at least in the near future this technology offers lowest CAPEX and time to deployment. On the other hand the capacity that copper can guarantee is not high, i.e., $100 \mathrm{Mbps}$ and can be provided for up to 400 meters reach [9]. In addition, according to Nielsen's law access bandwidth grows $50 \%$ per year. This means that, given the current traffic demand, VDSL2 will no longer be able to provide enough capacity for high-end customers in five years' time. On the other hand, until this limit will be reached it will be still reasonable to use as much as possible the existing copper infrastructure for backhauling indoor base stations. Higher capacity can be expected in ten years' time by using VDSL Bonding, Vectoring and G.Fast [9]. On the other hand this would also translate in a higher CAPEX since most of the existing equipment would have to be upgraded. This begs the question on whether this extra money would be better spent on additional fiber or microwave deployments, both offering more future proof solutions.

Microwave is quick and rather inexpensive to deploy compared to fiber. In the next ten years microwave appears as a good candidate to backhaul outdoor deployments thanks to its rather short time to deployment and relatively high capacity that can be provided (i.e., up to $10 \mathrm{Gbps}$ [3]). According to [14] microwave has the second highest CAPEX among the

\section{HETNET URBAN SCENARIO IN 2025}

Trying to predict how an urban HetNet deployment will look like in 2025 is not an easy task. There are a lot of variables at play including the type of HetNet deployment and the future performance outlook of each backhauling technologies. The scenario depicted in Figure 2 attempts to make such a prediction. It is an evolution of the assessment presented along with Figure 1, and it is based on the considerations made in Section III.

In the wireless deployment described in Figure 2, it can be noticed that the number of pico and femto base stations is increased compared to the scenario in Figure 1, resulting in a more dense base station deployment, especially indoor. However WLAN will still remain as an offload alternative for broadband traffic (e.g., one possibility would be to have operators deployed femto base stations for residential use with integrated WLAN capabilities). On the other hand macro base stations will remain, but with the old equipment being replaced by new $3 \mathrm{G}$ and $4 \mathrm{G}$ solutions providing coverage for GSM, EDGE, UMTS, and LTE. This is the consequence of the expected traffic increase in the mobile access segment.

As for the backhaul, the assumptions made in Figure 2 are based on the estimations presented in [1], where it is expected that in the next ten years the backhaul segment will comprise $50 \%, 44 \%$ and $6 \%$ of microwave, fiber and copper resources, 




Figure 2. Possible HetNet deployment with relative backhaul in a dense urban areas in year 2025.

respectively. The above estimation is also consistent with the technology outlook presented in Section III, where it was expected a gradual deployment of fiber as a replacement of the copper-based backhaul solutions almost everywhere (indoor for small base stations and outdoor for legacy $2 \mathrm{G}$ equipment). This migration from copper to fiber will guarantee a backhaul segment with more capacity, longer reach and better energy performance. Microwave on the other hand will still be popular thanks to its ability to provide enough bandwidth to meet the expected per-user broadband capacity requirement for at least the next ten years, i.e., about 300 Mbps per user [17]. Finally, in the next years copper will still be used in some regions as a legacy broadband connection for residential premises and used to piggyback backhaul traffic when fiber and microwave solutions are not available or still too costly to deploy (e.g., very remote rural areas).

\section{CONCLUSION}

This paper presents main challenges of future deployment of energy efficient backhauling for Heterogeneous Network (HetNet). The study is based on two HetNet deployment scenarios for dense urban areas, i.e., supporting traffic demand expected in 2015 and the traffic requirements envisioned for year 2025. It was found that in terms of energy efficiency fiber is the best option for backhauling heterogeneous networks traffic. However, the time for having a widely deployed fiber infrastructure can be quite long (i.e., also as a consequence of high (APEX). In the meantime copper that is already deployed almost everywhere can be a useful alternative for backhaul especially in the presence of indoor base station deployments. Microwave on the other hand represents the perfect compromise between CAPEX/OPEX, time to deployment, and capacity. For this reason it is expected that microwave will remain the preferred solution for backhauling outdoor base stations when their capacity requirements are not too high (i.e, micro and pico base stations) or when macro base stations cannot be reached by the fiber infrastructure.
In summary it is not possible to identify one unique backhaul solution able to fit all the requirements and satisfy all the needs. The "best" backhaul architecture will most probably be the result of a mix of microwave, fiber, and in rare cases, copper.

\section{ACKNOWLEDGMENTS}

The auktors would like to acknowledge Henrik Aspegren, Ulf Persson, and Henrik Åhlfeldt for the fruitful technical discussions and valuable input.

\section{REFERENCES}

[1] Koetz, S, "Mobile Breitband. heterogene mobilfunknetze und die Relevanz für regionalcarrier," Ericsson GmbH, Ericsson AB 2012.

[2] "Meeting the mobile backhaul chalienges." Transmode white paper, 2012.

[3] "Microwave Backhauling - Meeting the Capacity Challenges of 4G/LTE and Beyond," Ceragon technical brief, 2012.

[4] "Cisco Visual Networking Index: Global Mobile Data Traffic Forecast Update, 2012-2017," Cisco white paper, 2012.

[5] Tombez, S.; el al., "Impact of Backhauling Power Consumption on the Deployment of Heterogeneous Mobile Networks," Proc. IEEE CLOBECOM. 2011.

[6] Monti, P.: et al., "Mobile backhaul in heterogeneous network deployments: Technology options and power consumption," Proc IEEE ICTON, 2012.

[7] "Fiber, Microwave and Copper in the mobile backhaul, " SELTA Business case, Marke1 development, Nov. 2010, Ed. 2.

[8] "Mobile Backhaul: Fiber vs Microwave. " Cegragon white paper, 2009

[9] "DSL Acceleration: Making it Work." Alcatel Heavy Reading white paper, June 2012.

[10] Damnjanovic, A.; et al., "A survey on 3GPP heterogeneous networks," Wireless Communications, IEEE, 2011.

[11] Piro, G.; el al.. "HetNets Powered by Renewable Energy Sources: Sustainable Next-Generation Base stationular Networks," Internet Computing, IEEE, 2013.

[12] Tipmongkolsilp, O., et al., "The Evolution of Cellular Backhaul Technologies: Current Issues and Future Trends." Communications Surveys \& Tutorials, IEEE, 2011

[13] "Next-Generation Wireless Backhaul Solutions," BROCADE technical brief, 2009.

[14] "Cricial Economics for mobile data backhaul," Senza fili consulting white paper, 2011.

[15] "LTE backhauling Options and Archilectures." Miltelecom white paper, 2012.

[16] "Flexible Front and Backhauling for $4 G$ and Beyond," HFR FlexiHaul, Aug. 2012.

[17] "Consolidated requirements for European nexl-generation optical access networks," OASE project, D2.2.2, 2012. 